Acta Crystallographica Section D

Biological

Crystallography

ISSN 0907-4449

\title{
Development of an automated large-scale protein-crystallization and monitoring system for high-throughput protein-structure analyses
}

Protein crystallization remains one of the bottlenecks in crystallographic analysis of macromolecules. An automated large-scale protein-crystallization system named PXS has been developed consisting of the following subsystems, which proceed in parallel under unified control software: dispensing precipitants and protein solutions, sealing crystallization plates, carrying robot, incubators, observation system and image-storage server. A sitting-drop crystallization plate specialized for PXS has also been designed and developed. PXS can set up 7680 drops for vapour diffusion per hour, which includes time for replenishing supplies such as disposable tips and crystallization plates. Images of the crystallization drops are automatically recorded according to a preprogrammed schedule and can be viewed by users remotely using web-based browser software. A number of protein crystals were successfully produced and several protein structures could be determined directly from crystals grown by PXS. In other cases, X-ray quality crystals were obtained by further optimization by manual screening based on the conditions found by PXS.

₹ These authors contributed equally. $\S$ Present address: Institute for Protein Research, Osaka University, Yamadaoka 3-2, Suita,

Osaka 565-0871, Japan.

Correspondence e-mail: ryuichi.kato@kek.jp
(C) 2006 International Union of Crystallography Printed in Denmark - all rights reserved

\section{Introduction}

One of the major bottlenecks of high-throughput protein crystal structure determination is the crystallization process of protein samples. To solve this, many different proteincrystallization methods have been developed and a novel high-throughput approach has been proposed (Stojanoff, 2004). In order to increase the speed and the yield of protein crystallization, automated crystallization systems have been developed in recent years (Stevens, 2000; Sulzenbacher et al., 2002; Watanabe et al., 2002; Hui \& Edwards, 2003; Adachi et al., 2004; Shah et al., 2005). In addition, there are several commercially available protein-crystallization systems. For example, Oryx 6 (Douglas Instruments Ltd) can set up 96 wells in 12 min for sitting-drop vapour diffusion or the same number of wells in $50 \mathrm{~min}$ for microbatch. Cyberlab (Gilson Inc.) can automatically set up hanging drops but is not as fast as Oryx 6. The Hydra II Plus-One system (Matrix Technologies Co.) can dispense 96 sub-microlitre drops in $58 \mathrm{~s}$ and set up crystallization plates semi-automatically. Simultaneously, several laboratories and structural genomics projects have developed original crystallization systems. In the case of Syrrx Inc., the system can set up 2880 drops for vapour diffusion per hour (Hosfield et al., 2003). To date, this setup speed is the
Received 12 March 2006 Accepted 21 June 2006 
Table 1

Specifications of the high-throughput automated crystallization system PXS.

\begin{tabular}{cl}
\hline $\begin{array}{c}\text { Dispensing part } \\
\text { Crystallization method }\end{array}$ & Sitting-drop vapour diffusion \\
Dispensing method & Air displacement with disposable tips \\
Speed of setting up & 80 plates $(96$-well) per hour \\
crystallization drops & 7680 drops per hour \\
Dispensing volume & $0.5 \mu$ l protein sample $+0.5 \mu l$ precipitant \\
Scale of one batch of & 80 plates $(96$-well) \\
plates & 7680 drops \\
& $(8$ protein samples) \\
& (960 crystallization conditions) \\
Sealing method & Adhesive seal \\
Observation part & Average 80 s per plate $(96-w e l l)$ \\
Observation speed & Color $640 \times 480$ pixels $(3 \times$ colour CCD camera) \\
Images & Three selectable magnifications $(\times 1, \times 1.5, \times 6)$ \\
& Polarized light optical system \\
Light source & High-brilliance white LED array \\
Image data server & Storage capacity 1 TB \\
& Linux-based web server \\
Incubator & 1100 plates per system \\
& 350 (or 200) plates per incubator \\
& Temperature $281-298( \pm 0.5) \mathrm{K}$ \\
&
\end{tabular}

fastest in the world. The Hauptmann-Woodward group has developed a microbatch system which dispenses protein and precipitant solutions and oil (Luft et al., 2001). The TERA system that has been developed by RIKEN in Japan also selected the microbatch method (Sugahara \& Miyano, 2002). It can set up 2304 drops per day. To achieve high throughput, almost all the systems adopt computer-controlled robots and unified software to manage the vast amount of data, including crystallization conditions and images of crystallization drops.

Synchrotron X-ray crystallography is one of the most powerful techniques to determine three-dimensional structures of biomolecules. However, as structural scientists attempt to solve substantially more difficult and complex structures, it becomes harder to obtain crystals of sufficient size. In such a situation, a high-throughput crystallization system will be important to screen thousands of crystallization conditions automatically and to obtain crystals of sufficient quality for structure determination.

\section{System description}

The following three points needed to be addressed in order to develop an automated crystallization system for our structural proteomics project (Japanese National Project on Protein Structural and Functional Analyses; Protein 3000). Firstly, we chose the sitting-drop vapour-diffusion method for the crystallization procedure for the following reasons. We are planning to develop an overall system which consists of crystallization, observation and evaluation of crystallization drops, harvesting crystals from drops, replacement of mother liquor with cryoprotectant solution and freezing crystals in liquid nitrogen. Automated micro-hands, which are now under development in collaboration with the group of Dr Tanikawa (Tanikawa \& Arai, 1999), must be able to access crystals in the drops. The microbatch method was excluded since it is very difficult to pick up crystals through the layer of oil. The sitting- drop vapour-diffusion method is more suitable for highthroughput operation than the hanging-drop method, which needs additional manipulation steps to flip coverglasses upside down. Secondly, the setup speed should be faster than that of manual handling. Thirdly, contamination of solutions should be avoided as much as possible.

To realise these goals, we developed a fully automated crystallization system named PXS, which includes a crystalobservation subsystem and computer storage for crystal images. It consists of six parts, (i) dispensing system for precipitants and protein solutions, (ii) sealing system, (iii) carrying robot, (iv) incubators, (v) observation system and (vi) image-storage server (Fig. 1), which are controlled by unified control software. During the development of PXS, we were careful to address the following three points: to minimize mechanical vibrations as much as possible, to avoid contamination during preparation of drops and to achieve the highest speed for dispensing and observation. PXS can set up 7680 drops from a matrix of 960 precipitants and eight proteins in one processing cycle and can finish the cycle within $1 \mathrm{~h}$, including the time to set up and replenish supplies such as crystallization plates and tips. The specifications of PXS are summarized in Table 1. Movies of the motion are available at http://pfweis.kek.jp/system/PXS.html.
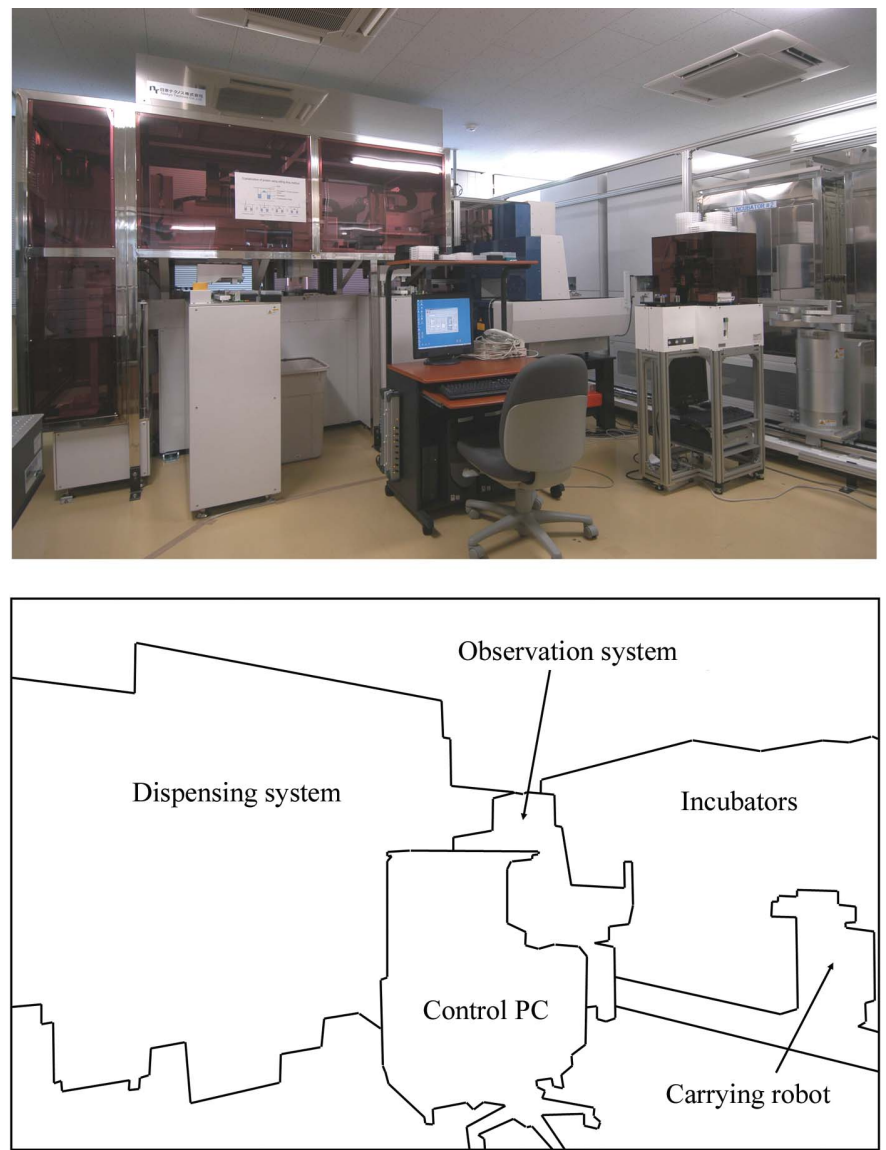

Figure 1

Overall view of the automated large-scale protein crystallization and monitoring system PXS. 


\subsection{Crystallization plate}

A new 96-well crystallization plate, named a KEK crystallization plate, was designed specifically for PXS (Fig. 2). It conforms to the ANSI/SBS standards published by the Society for Biomolecular Screening (http://www.sbsonline.org/msdc/ approved.php). It was designed for sitting-drop vapourdiffusion crystallization. At the centre of each well, there is a tower on which a drop consisting of a mixture of protein and precipitant solutions can be placed. The position of the tower is located at the centre of the well for ease of manipulation and harvesting of crystals. The crystallization drop should be able to be handled by an automated micro-hand since harvesting crystals is a critical part of our fully automated unified system, which will eventually cover steps from protein production to data collection.

The total volume of each well is about $400 \mu \mathrm{l}$ and the maximum dispensing volume of precipitant solution is about $300 \mu \mathrm{l}$. The roof of the tower is smoothly planed and the surrounding edge rises to prevent spillage of drop solution from the top of the tower. The maximum volume of the mixture of protein and precipitant solutions is about $10 \mu \mathrm{l}$ for each tower. Usually, the volume of the drop is $1 \mu \mathrm{l}$ as a mixture of $0.5 \mu \mathrm{l}$ of both solutions. Each plate is identified by a barcode label on the side.

\subsection{Dispensing system}

In order to achieve high throughput and no crosscontamination, the following two points were considered in the development of the dispensing system. Firstly, the

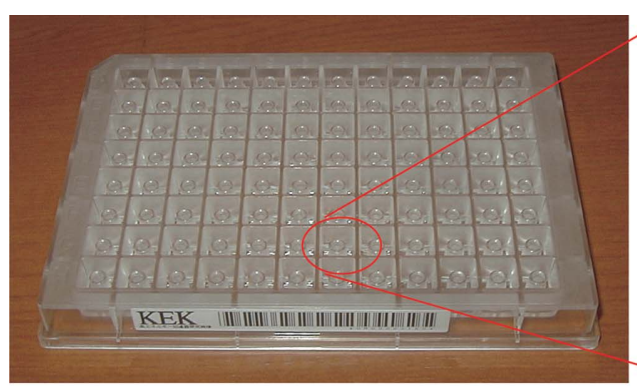

(a)

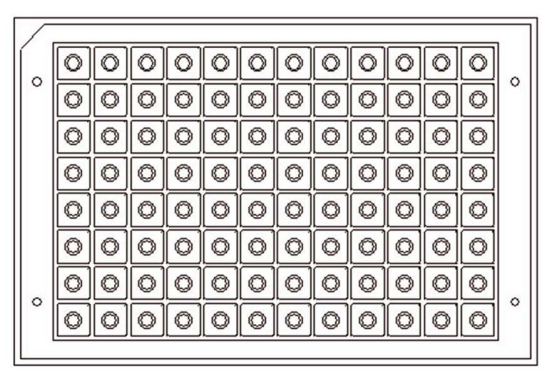

(c)

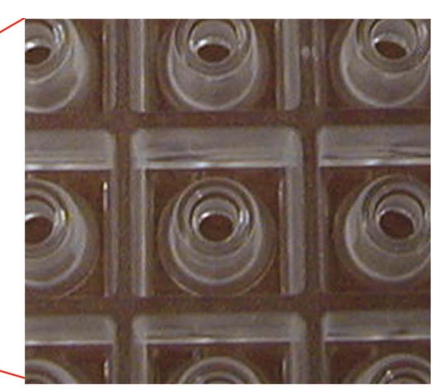

(b)

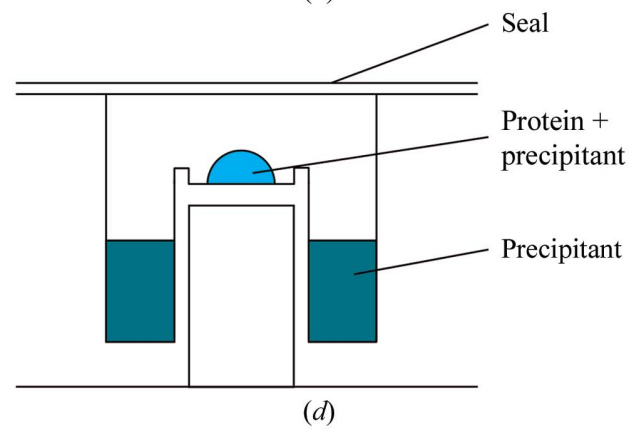

Figure 2

Crystallization plate developed de novo for PXS. The outer dimensions and the arrangement of the wells conform to the SBS standard for 96-well plates. A crystallization drop is dispensed onto the top of the tower at the centre of the well and precipitant solution is poured into the moat around the tower. (a) Overall view, $(b)$ close-up view and $(c)$ a projection of the crystallization plate. $(d)$ Schematic drawing of a sitting-drop crystallization procedure using the plate. dispensing system has a total of four independent dispensers controlled simultaneously. The parallel processing of dispensing solutions allows a substantial decrease in the total time for high-throughput operation. Secondly, disposable tips were used. Compared with other dispensers which have fixed nozzles and do not exchange dispensing tips, our dispensing system has two advantages. Since the time required for washing and drying the fixed nozzles is substantially longer than that for exchanging disposable tips, disposable-tip systems are faster. Moreover, cross-contamination never occurs. Aspiration and discharge of solutions are performed by the air-displacement method in all dispensers. All four dispensing heads can move with three degrees of freedom ( $X, Y$ and $Z$ axes) to access various dispensing points on the crystallization plate.

The dispensing system consists of four parts. The first part is a crystallization-plate supplier (Fig. 3a). Plates are stored in two plate stackers whose capacity is 40 plates each. The plate supplier can supply a total of 80 plates in a row. At the top of the plate supplier, there is a barcode reader which reads plate IDs into the system software and the database. The next part is dispenser 1, with 96 syringes for precipitant solutions (Fig. 3b). With 96 disposable tips which are automatically supplied by a tip stocker, it dispenses 96 precipitant solutions from a deep well plate to the crystallization plate in a non-contact manner in one step. A total of ten deep well plates, which contain precipitants made in-house or purchased commercially, can be set up on the turntable. The standard volume of precipitant solution is $180 \mu \mathrm{l}$ in each crystallization well. The third part is dispenser 2 for dispensing protein solutions (Figs. $3 c$ and $3 d$ ).

This part has a sample holder and two sets of dispensers: dispenser $2 \mathrm{~A}$ and dispenser 2B. The sample holder can store eight $0.5 \mathrm{ml}$ Eppendorf tubes containing different protein solutions at $277 \mathrm{~K}$ controlled by a Peltier cooling system. In order to realise highthroughput operation, the protein solution is first dispensed into eight wells in a middle plate, which has 96 V-shaped wells, by a single-syringe dispenser 2A with contact dispensing (Fig. 3c). The solutions from the eight wells are then dispensed to the crystallization plate by an eight-syringe dispenser $2 \mathrm{~B}$ with contact dispensing (Fig. 3d). The required sample volume for one 96-well plate with a $0.5 \mu 1$ dispensing volume is $52 \mu \mathrm{l}$. Thus, the dead-volume of the sample is $4 \mu \mathrm{l}$ per plate $(8 \%)$. Finally, drops are made by mixing precipitant and protein solutions. 96 syringes of dispenser 3 aspirate 96 precipitant solutions from the reservoirs simultaneously, move to the top of the tower where protein solutions have been already dispensed by dispenser 2 , 
discharge the precipitant solutions in a contact manner and then mix them by repeated aspiration and dispensing several times (Fig. 3e).

Each part of the dispensing system works simultaneously and all of them are connected by a shuttle transporting system (see supplementary material $\mathbf{1}$ ). The crystallization plates are transported from the plate supplier to dispensers 1,2 and 3 and then to the transfer port by the shuttle system in this order. The combination of the multichannel dispensing syringes, disposable tip system and parallel processing assures high throughput. The dispensers and the plate-transportation

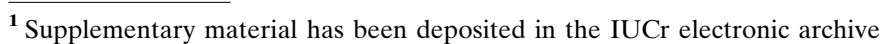
(Reference: CY5038). Services for accessing this material are described at the back of the journal. system are covered by a clean booth with a HEPA filter to prevent contamination with dust.

The reproducibility and accuracy of the dispensers were measured as follows. Dispenser 1 was tested using three solutions (containing $10 \mathrm{mM}$ sodium phosphate buffer $\mathrm{pH}$ 7.0) with different viscosities: water, 30\% 2-propanol and 30\% PEG 8000 . The reproducibility was calculated from the absorbance of each solution containing $0.0005 \%$ bromophenol blue (BPB). The absorbance of the solutions in each well was measured by a plate spectrophotometer (Iwaki, EZS-ABS). Since the typical dispensing volumes of dispensers 2 and 3 are too small to be measured by the procedure used for dispenser 1 , we used an alternative procedure. $0.5 \mu \mathrm{l}$ of $0.4 \%$ BPB solutions with different viscosities were dispensed onto the KEK crystallization plate by dry dispensing and diluted with
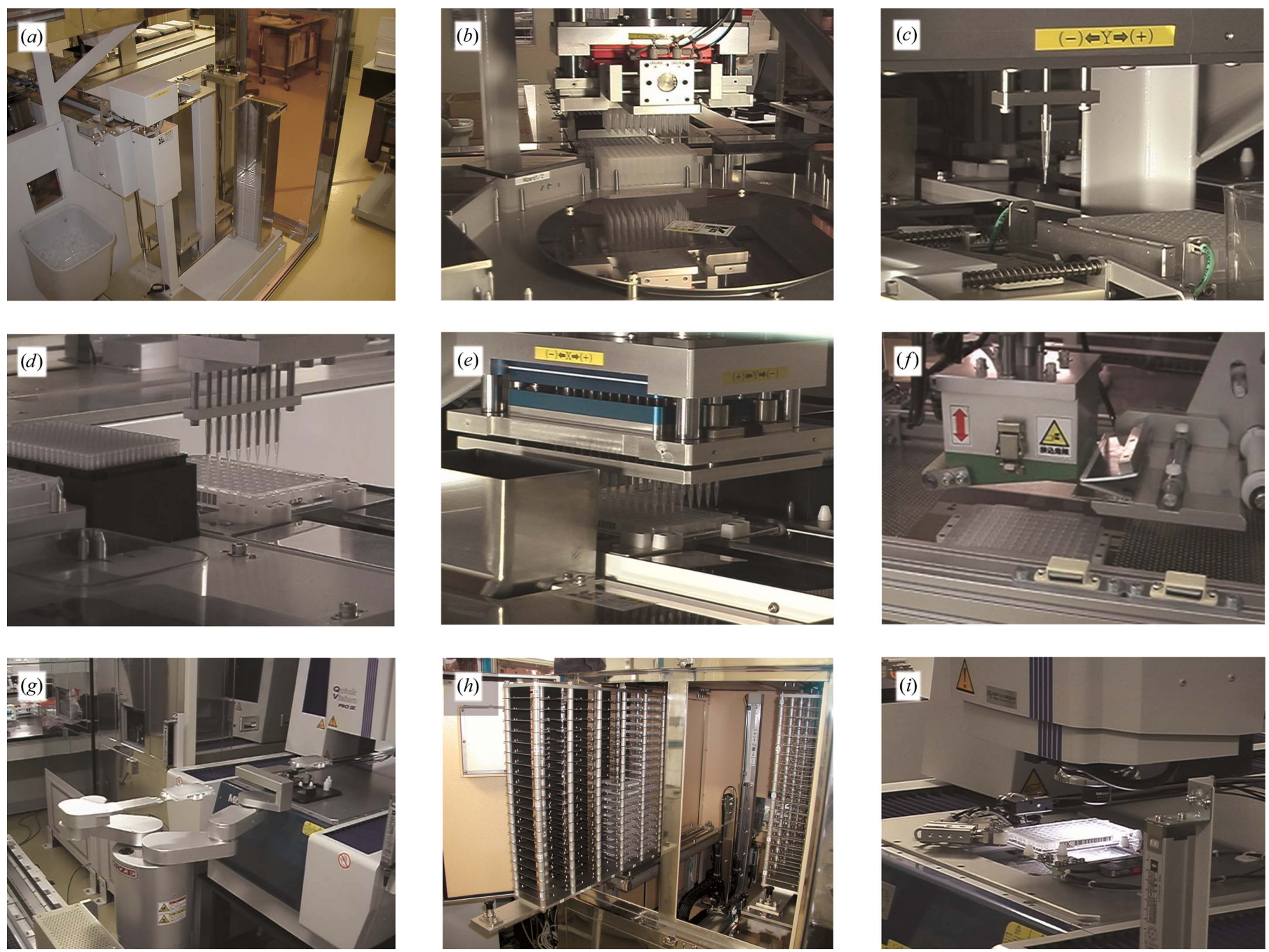

Figure 3

Process of the large-scale protein crystallization and monitoring system PXS. (a) Crystallization plate supplier. (b) Dispenser 1. Deep-well plates containing precipitant solution are placed on the turntable. The dispensing head with 96 disposable tips is seen in the centre. (c) Dispenser 2 . The first dispensing head with one disposable tip dispenses protein solution from one Eppendorf tube to eight wells on a middle plate seen on the right. $(d)$ The second dispensing head of dispenser 2 with eight disposable tips. (e) Dispenser 3 with 96 disposable tips aspirates precipitant solutions from 96 wells and mixes them with protein drops on the crystallization plate. $(f)$ The sealer. $(g$ ) Carrying robot and observation system. The carrying robot with two arms improves the total throughput of the system. $(h)$ Inside of the incubator. There is an automatic double door for importing and exporting crystallization plates at the opposite side of the emergency door, which is open to give a clear view of the inside. (i) Close-up view of the observation system. The crystallization plate is illuminated from underneath and an image of each drop is acquired by the colour CCD camera above the plate. The plate is fixed on the stage and both the light source and the camera move synchronously to take images of the 96 wells. 
Table 2

Accuracy of dispenser 1 .

\begin{tabular}{lllc}
\hline Volume $(\mu \mathrm{l})$ & Solution & $\mathrm{CV} \dagger(\%)$ & Accuracy $¥(\%)$ \\
\hline \multirow{2}{*}{100} & Water & 1.4 & 98 \\
& 30\% 2-propanol & 1.7 & 101 \\
\multirow{3}{*}{180} & 30\% PEG 8000 & 2.6 & 97 \\
& Water & 2.1 & 98 \\
& 30\% 2-propanol & 1.3 & 97 \\
& 30\% PEG 8000 & 6.6 & 92 \\
\hline
\end{tabular}

$\dagger$ Coefficient of variation. The value is an average of four measurements of each $\mathrm{CV}$ value calculated from 96 wells. $\ddagger$ The value is an average over all 96 wells and four measurements.

Table 3

Accuracy of dispensers 2 and 3 at a dispensing volume of $0.5 \mu \mathrm{l}$.

\begin{tabular}{llll}
\hline Dispenser & Solution & CV $\dagger(\%)$ & Accuracy $¥$ (\%) \\
\hline Dispenser 2 & Water & 4.6 & 92 \\
& 30\% 2-propanol & 4.5 & 86 \\
Dispenser 3 & 30\% PEG 8000 & 6.0 & 92 \\
& Water & 7.3 & 89 \\
& 30\% 2-propanol & 1.7 & 86 \\
& 30\% PEG 8000 & 4.7 & 89 \\
\hline
\end{tabular}

$\dagger$ Coefficient of variation. The value is an average of five measurements of each CV value calculated from 96 wells. $\$$ The value is an average over all 96 wells and eight measurements.

$180 \mu \mathrm{l} 10 \mathrm{~m} M$ sodium phosphate buffer $\mathrm{pH} 7.0 .120 \mu \mathrm{l}$ of the solutions were transferred to standard SBS plates and the absorbance was measured by the plate spectrophotometer. The accuracy of dispensing was calculated by weighing the plates from a number of independent trials. As summarized in Tables 2 and 3, dispensers 1, 2 and 3 can dispense solutions with different viscosities accurately and reproducibly.

\subsection{Sealing material and system}

Immediately after making drops in the crystallization plate, the top of the plate must be sealed. In this step, heat cannot be used because it may affect the protein. The sealing material must be clear because observation of the drops is carried out through the sealing material using an observation system above the plate. In addition, the vapour permeability of the sealing material should be as low as possible. We developed a new sealing material which satisfies the above requirements. The sealing material is transparent and does not polarize light. As shown in the supplementary material, the permeability of the sealing material is the same as available commercial crystallization films. The transparency and adhesiveness of the film and glue do not change after brief contact with the precipitant solutions. During a $1 \mathrm{y}$ incubation at $293 \mathrm{~K}$, the transparency of the sealing materials did not change and the film did not peel off the crystallization plate (data not shown).

The sealing system receives the crystallization plate from the dispensing system, seals its top with the adhesive seal (Fig. 3f) and then transfers it to the carrying robot. One roll of the sealing film can cover 3000 crystallization plates. The sealing system is also covered by a clean booth with a HEPA filter to prevent contamination with dust.

\subsection{Carrying robot}

After sealing the crystallization plates, the carrying robot transports them to the incubators or to the observation system (Fig. $3 g$ ). The carrying robot also shuttles the crystallization plates between the incubators, the observation system, a plate I/O port and a plate trash port. To realise both high speed and minimum vibration during transport, a linear motor-driven two-armed robot of the kind that is used in the semiconductor industry for transporting wafers with minimal vibration was installed. Owing to the double arm, the plate-exchanging time is about halved compared with a single-arm robot.

\subsection{Incubators}

The crystallization plates are stored in incubators. We developed two kinds of incubators with different capacities: 200 and 350 plates (Fig. 3h). The size of the incubator is dictated by the limit on access time of the plate in and out of the incubator; the transporting arm grabs the plate at the entrance door, brings it to a shelf and returns to the door to receive the next plate. If the time is longer than that of the dispensing system, the incubator becomes a bottleneck which reduces the total throughput of the whole system. At present, four incubators have been installed and the total storage capacity reaches 1100 plates. The incubators have double automatic doors for import and export of the plates to prevent temperature disturbance in the incubators. The temperature of each incubator can be set independently between 281 and $298 \mathrm{~K} \pm 0.1 \mathrm{~K}$. We measured the time course of temperatures at eight different positions in the incubator using thermocouples. In the initial half hour after turning on the switch of the incubator, a slight temperature drift was observed (see supplementary material). This temperature fluctuation only occurred upon the initial drift and was never observed again during two weeks of experiment (data not shown). The uniformity of the temperatures in the incubator is maintained within $\pm 0.5 \mathrm{~K}$ at the eight different positions for which it was measured.

To prevent unwanted vibrations, the storage shelves are fixed firmly, the plate-delivery robot with four degrees of freedom $(X, Y, Z$ axes and rotation) is mounted in the incubator using elastic vibration isolators and a cooling unit for temperature control is physically isolated from the frames of the shelves. A barcode reader, which recognizes each crystallization plate, is installed at the door. The ID codes and positions of the plates in the shelves are stored in the database of the incubator itself. The data are copied to the main database server and updated to synchronize them. As described below in detail in $\$ 2.7$, PXS is designed to be modular and several aspects of the system, including the incubator number and size, are easily expandable. We can incorporate additional incubators when the storage space for crystallization plates is filled.

\subsection{Observation system}

Observations of crystallization drops are scheduled by users using the control software. The observation system takes 
photo images automatically according to the schedule. To prevent vibration of crystallization drops, each plate is fixed during observation. An observation head with a colour CCD camera moves along the $X, Y$ and $Z$ axes to take images of drops from above (Figs. $3 g$ and $3 i$ ). It takes about $80 \mathrm{~s}$ to complete observation of one plate ( 96 wells). The light source, which is located below the plate, moves synchronously with the observation head. By replacing the prototype halogenlamp fibre optics with a highly brilliant white LED array, the quality of the images was improved drastically. The observation system has three different magnifications $(1 \times, 1.5 \times$ and

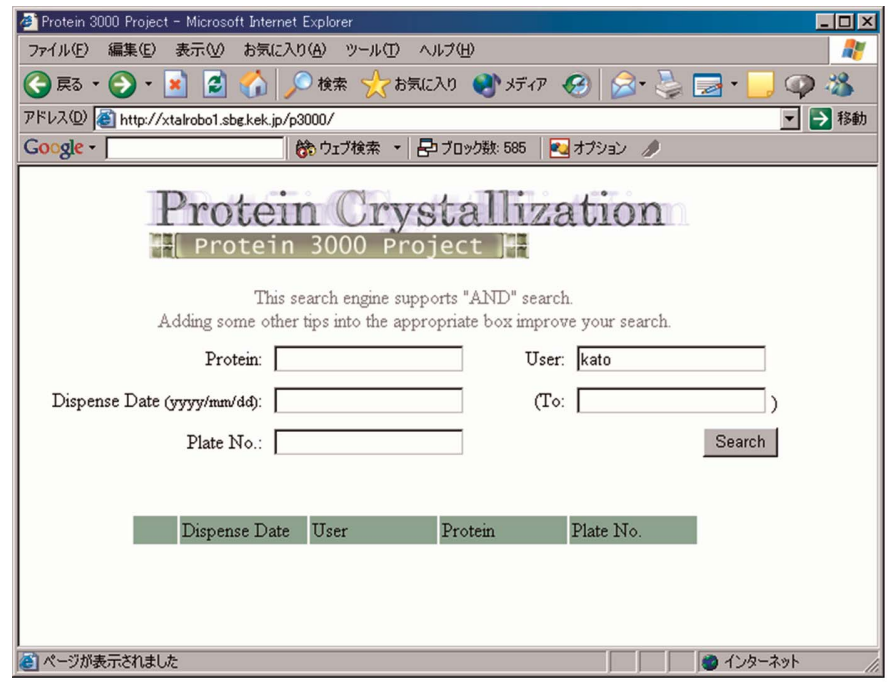

(a)

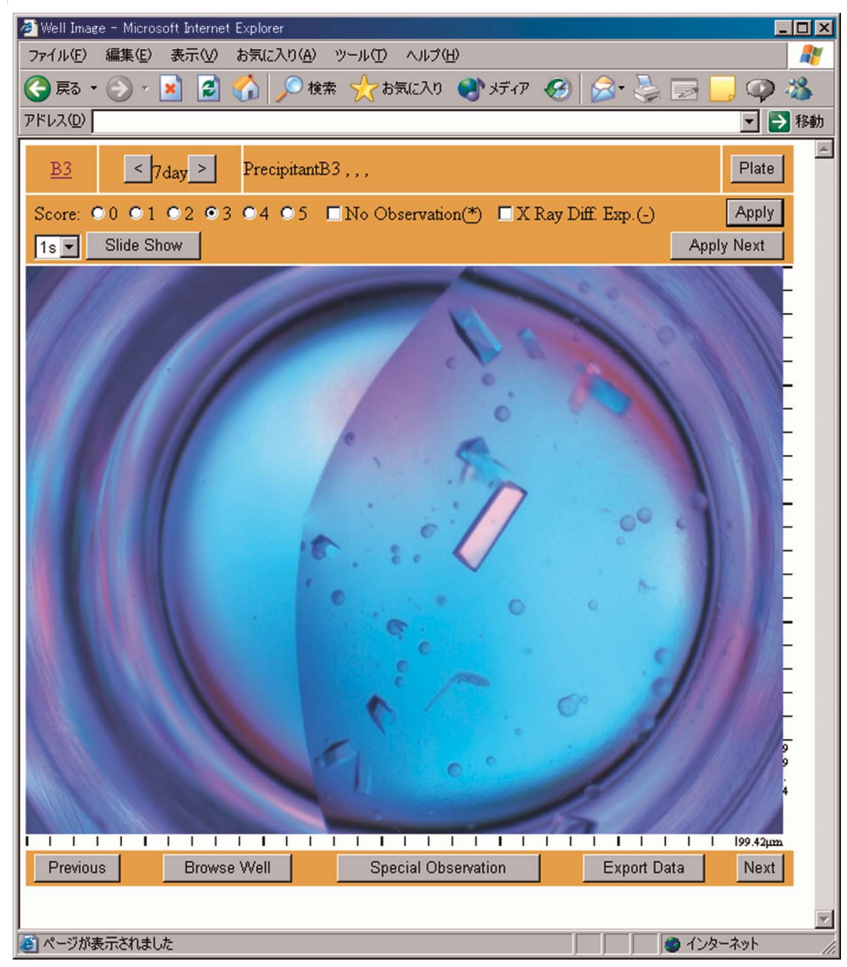

(c)
$6 \times)$, a combination of a fixed-angle polarizer and a rotatable analyzer, variable brightness control of the light source, adjustable gain of the CCD camera and pan-focus image generation from multiple sliced images. All of these parameters can be set up by the control software. As part of the preparation procedure, users are requested to input an observation schedule including those parameters into the control software. The observation parameters can be changed even during a scheduled observation procedure. The PXS is located in a room roughly maintained at $293 \mathrm{~K}$ using air conditioning. Since the incubators are now used at $293 \mathrm{~K}$, only

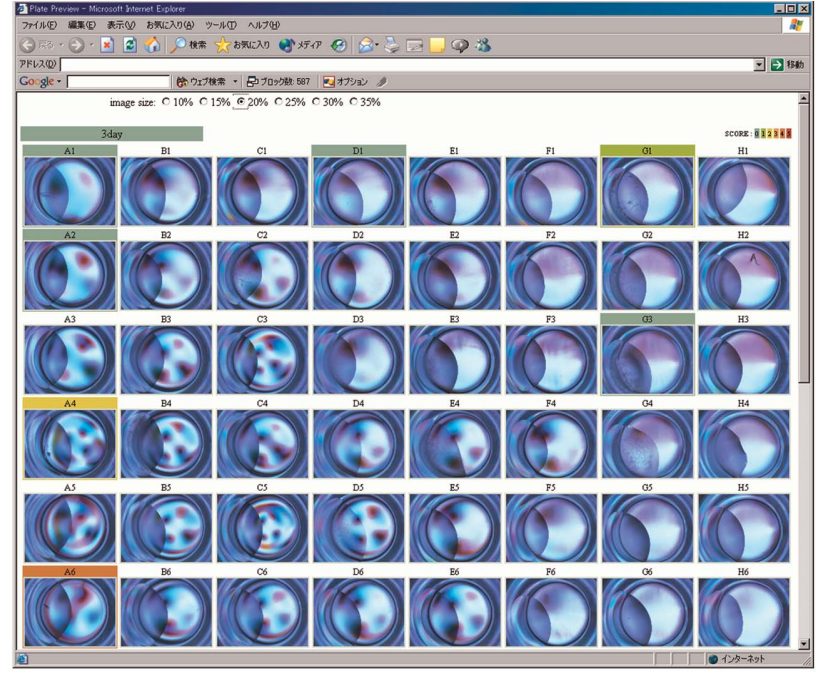

(b)

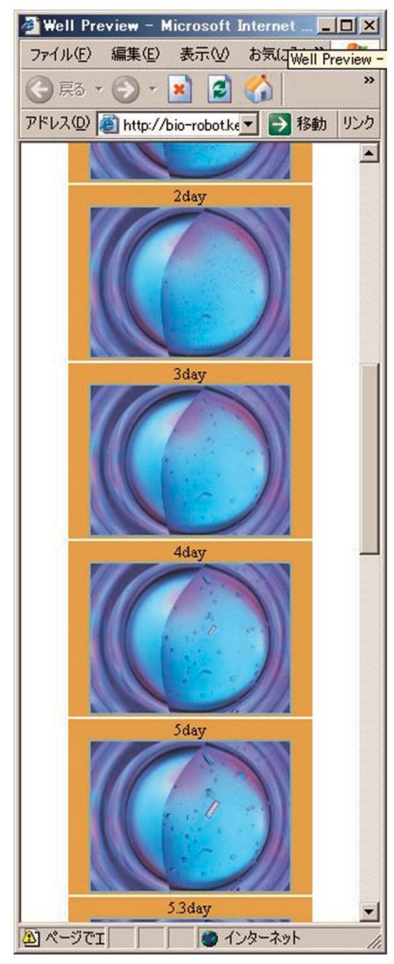

(d)

Figure 4

Viewing software. Users can view images of the crystallization plates any time using a web browser. (a) Login window. (b) Browser images of 96 drops. (c) An expanded image of a drop. (d) Chronological images of one drop. 
negligible temperature changes occur during crystallizationplate transportation and observation. We plan to develop and install a new incubator which contains a built-in observation system.

Users can check the images using a Java script running a web browser remotely at any time. Image data are compressed into JPEG format and stored in the database. Users can search their crystallization plates by sample name, user name, dispensed date or plate ID (Fig. $4 a$ ) and inspect all 96 images of the selected plate for the selected observation date in a new window (Fig. 4b), as well as expanded images (Fig. 4c) and chronological developments (Fig. 4d) in the crystallization drops. They can also obtain detailed information such as sample name, user name, dispensed date, operator name, plate ID, precipitant ID, observation schedule, composition of the precipitant, well position and any other remarks by clicking corresponding buttons in the graphical user interface (GUI). Users can check the drops, judge whether they contain crystals, precipitation or clear solution and score them, which can be recorded in the database. A schematic representation of the image-data flow is shown in the supplementary material.

\subsection{Control software}

The system-control software is written in a modular architecture (see supplementary material). The motions of the plate supplier and dispenser 1 are controlled by one personal

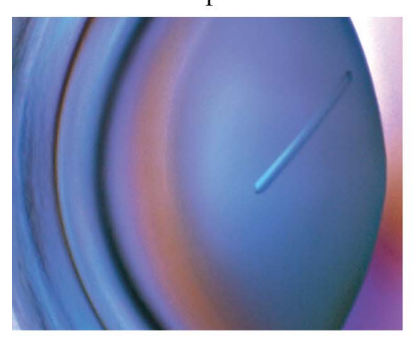

$3 b$

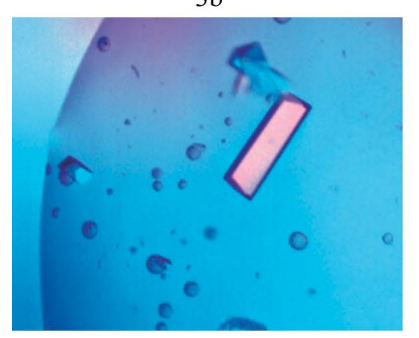

6

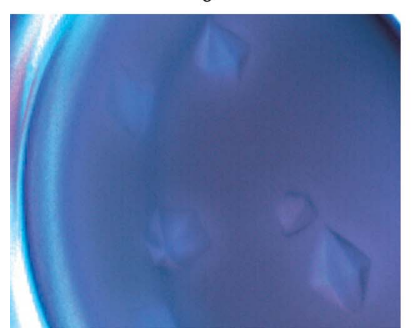

$2 \mathrm{a}$

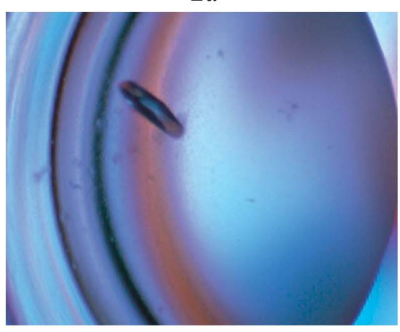

4

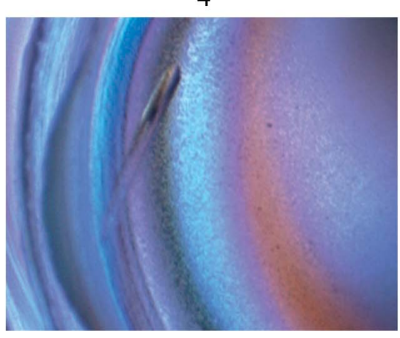

7

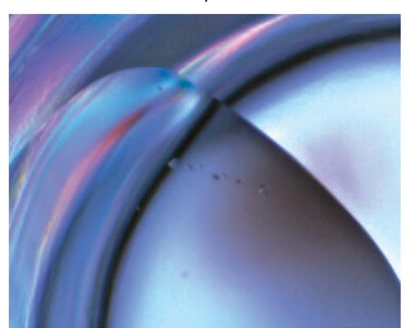

computer (PC) and dispensers 2 and 3 by another PC. The observation system has its own PC and software with interfaces to communicate with other software and to export image data. A total of six modular applications are run in the main control PC: (i) a main GUI module for communication with the control PCs of the plate supplier, dispensers 1,2,3, the database server and the subsequent applications, (ii) shuttletransporting system and sealing-system control module, (iii) incubator control module, (iv) carrying robot control module, (v) plate I/O port control module and (vi) observation-system control module. Every control module controls its corresponding system via digital I/O, Ethernet or RS232C interfaces. The communications between the main GUI module and the other modules are via the inter-application communication protocols XML and SQL. The modular architecture is designed not only at the software level but also at the hardware level allowing easy upgrades of the system components such as incubators, observation system and, in particular, future dispensing systems.

\section{Examples of crystallization trials}

The crystallization system PXS was first tested with several proteins that were known to crystallize. As an initial set of trials, the following commercially available 480 precipitants were used: Crystal Screen (Jancarik \& Kim, 1991), Crystal Screen 2, PEG/Ion Screen, Natrix and MembFac from

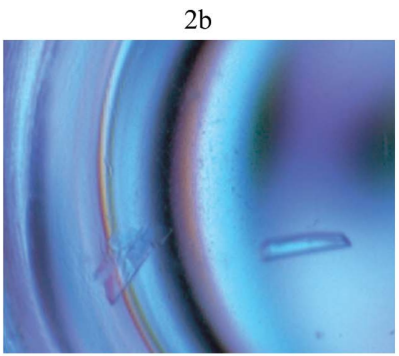

$5 a$

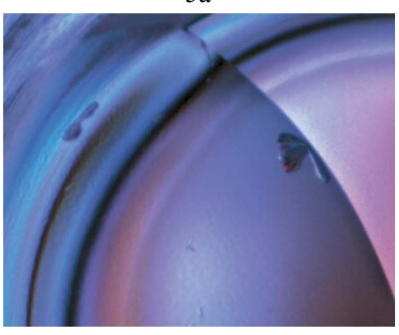

8

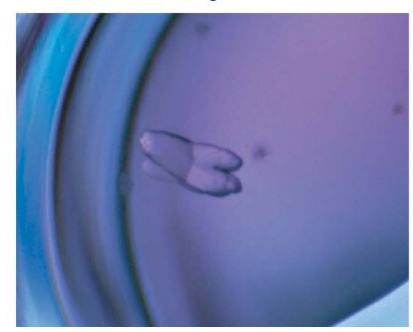

$3 a$

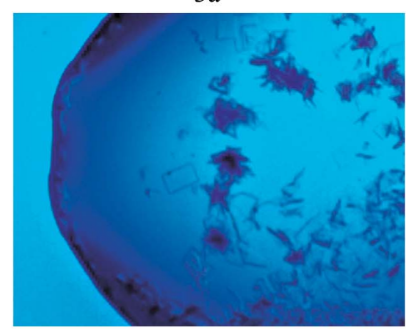

$5 \mathrm{~b}$

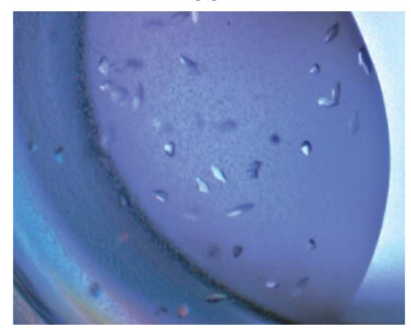

$1 \mathrm{~mm}$

Figure 5

Examples of protein crystals produced by PXS. The preliminary diffraction statistics of the crystals are summarized in the supplementary material. 
Hampton Research, Wizard I, Wizard II, Cryo I and Cryo II from Emerald BioSystems Inc. and Stura Footprint Screen (Stura et al., 1992) from Molecular Dimensions Ltd. A typical crystallization condition contained $0.5 \mu \mathrm{l}$ of $5-10 \mathrm{mg} \mathrm{ml}^{-1}$ protein solution mixed with $0.5 \mu \mathrm{l}$ precipitant solution and a $180 \mu \mathrm{l}$ reservoir volume and the crystallization plates were stored at $293 \mathrm{~K}$. We first tested the standard crystallization protein samples lysozyme (Sigma), glucose isomerase (Hampton Research) and xylanase (Hampton Research) and succeeded in obtaining crystals in all cases (data not shown). We then tested several other proteins which have been shown to produce crystals by manual crystallization procedures. For example, a human sialidase Neu2 (Chavas et al., 2005) and other proteins (manuscripts in preparation) successfully produced crystals under the same conditions as the previously described manual crystallization.

Next, we carried out crystallization experiments of new protein samples for de novo structural determination using PXS. In many cases, small or multiple crystals that were not suitable for diffraction data collection were obtained using the initial 480 screening conditions. For cases where no crystals were obtained, additional screenings were carried out by changing the crystallization conditions, such as the protein concentration or crystallization temperature, and by the addition of small compounds to the protein solution. Improvement of the crystals by further optimization led to well diffracting crystals and to successful structure determination. In some cases, we could obtain X-ray-quality single crystals from the initial crystallization trials (Fig. 5) and could solve the structures. The preliminary diffraction data are summarized in the supplementary material. One of the detailed structures has been published (Satoh et al., 2006) and the remaining structures will be reported in the near future.

\section{Conclusions}

To solve one of the bottlenecks of protein crystallography, an automated large-scale protein-crystallization system named PXS has been developed. PXS has shortened the time required for crystallization step by more than 100-fold compared with manual crystallization. Users can view images of the crystallization drops remotely using a web browser. We successfully produced a number of crystals from various proteins using PXS. In some cases, we solved their crystal structures without further optimization of crystallization conditions. The development and use of PXS was supported by the Protein 3000 project, with the goal of pursuing structural and functional analyses of proteins. The system is located at the Structural Biology Research Center, KEK-PF and is used by about 30 people in the centre at the present time. We are planning to open PXS to users outside the centre after resolving present issues with incubator capacity and the cost of supplies.
To improve the system, we are developing a high-speed observation system with 96 lenses and CCD cameras bundled in parallel to capture images of all 96 drops of a crystallization plate in tenths of a second (patent pending). In addition, we plan to develop a nanolitre dispensing system, which will reduce sample volume and dispensing time, and an automated crystal scoring system based on various image-processing techniques. As a longer term development project, we also plan to develop a fully automated system which encompasses the whole range of crystallographic preparation steps: sittingdrop preparation, observation, scoring, harvesting, soaking with a cryoprotectant and freezing crystals.

We are grateful to Mr T. Usui (Nikkyo Technos Co. Ltd), Mr Y. Yamasaki (IS Technology Inc.), Mr M. Ashida (DSD Ltd) and Mr T. Ikeda (Japan Support System Ltd) for crucial cooperation in this work. We also would like to thank the following Japanese companies for their contributions in the construction of PXS: Nikkyo Technos Co. Ltd (Tokyo), IS Technology Inc. (Ibaraki), DSD Ltd (Tokyo), Japan Support System Ltd (Ibaraki), Mitutoyo Corporation (Kanagawa), Corona Giken Ltd (Chiba), Shanti Corporation (Tokyo), Dan Science Co. Ltd (Tokyo), Rorze Corporation (Hiroshima) and Lintec Ltd (Tokyo). This work was supported by the Protein 3000 project of the Ministry of Education, Culture, Sports, Science and Technology of Japan (MEXT).

\section{References}

Adachi, H., Takano, K., Matsumura, H., Niino, A., Ishizu, T., Inoue, T., Mori, Y. \& Sasaki, T. (2004). Jpn J. Appl. Phys. 43, L76-L78.

Chavas, L. M., Tringali, C., Fusi, P., Venerando, B., Tettamanti, G., Kato, R., Monti, E. \& Wakatsuki, S. (2005). J. Biol. Chem. 280, 469-475.

Hosfield, D., Palan, J., Hilgers, M., Scheibe, D., McRee, D. E. \& Stevens, R. C. (2003). J. Struct. Biol. 142, 207-217.

Hui, R. \& Edwards, A. (2003). J. Struct. Biol. 142, 154-161.

Jancarik, J. \& Kim, S.-H. (1991). J. Appl. Cryst. 24, 409-411.

Luft, J. R., Wolfley, J., Jurisica, I., Glasgow, J., Fortier, S. \& DeTitta, G. T. (2001). J. Cryst. Growth, 232, 591-595.

Satoh, T., Sato, K., Kanoh, A., Yamashita, K., Yamada, Y., Igarashi, N., Kato, R., Nakano, A. \& Wakatsuki, S. (2006). J. Biol. Chem. 281, 10410-10419.

Shah, A. K., Liu, Z.-J., Stewart, P. D., Schubot, F. D., Rose, J. P., Newton, M. G. \& Wang, B.-C. (2005). Acta Cryst. D61, 123-129.

Stevens, R. C. (2000). Curr. Opin. Struct. Biol. 10, 558-563.

Stojanoff, V. (2004). Structure, 12, 1127-1128.

Stura, E. A., Nemerow, G. R. \& Wilson, I. A. (1992). J. Crystal Growth, 122, 273-285.

Sugahara, M. \& Miyano, M. (2002). Tanpakushitsu Kakusan Koso, 47, 1026-1032.

Sulzenbacher, G. et al. (2002). Acta Cryst. D58, 2109-2115.

Tanikawa, T. \& Arai, T. (1999). IEEE Trans. Robot. Autom. 15, 152-162.

Watanabe, N., Murai, H. \& Tanaka, I. (2002). Acta Cryst. D58, 15271530. 